\title{
Los noticieros espejo o la liviandad de la información
}

\author{
(a) \\ Liliana Gutiérrez-Coba \\ Juan Carlos Gómez-Giraldo \\ Juan Camilo Hernández-Rodríguez \\ Germán Arango-Forero \\ Recibido: 15 de diciembre de 2010 \\ Aprobado: 6 de febrero de 2011
}

\begin{abstract}
Resumen
Este artículo explora las implicaciones de la falta de contextualización de la información que publican los noticieros de televisión colombianos en la toma de decisiones y la formación de opinión pública en los ciudadanos. Está basado en los resultados de una investigación realizada por el Observatorio de Medios de la Facultad de Comunicación de la Universidad de La Sabana y el Centro Multimedial de la Universidad Eafit, y financiada por la Comisión Nacional de Televisión que, a través de un análisis de contenido, indaga sobre la estructura, cobertura y contenidos de los noticieros de la televisión en Colombia. El predominio de la noticia escueta, sin contexto o con pocos elementos de éste, termina por desdibujar la función orientadora del periodismo.
\end{abstract}

Palabras clave: Televisión, noticieros, información, contextualización.

Investigadores del Observatorio de Medios y del Grupo de Investigación en Periodismo, GIP, de la Universidad de La Sabana. E-mail: liliana.gutierrez1@unisabana.edu.co; juan.gomez@unisabana.edu.co,juan.hernandez6@unisabana.edu.co,german.arango@unisabana. edu.co. 


\title{
Mirror news or information frivolity
}

\begin{abstract}
This article explores implications of the lack of contextualization of the information disclosed by Colombian television news on citizens' decision making and creation of public opinion. The articles is grounded on results obtained from a research conducted by the Media Observatory of Universidad de La Sabana School of Communication and the Multimedia Center of Universidad EAFIT, sponsored by the National Television Commission which, through a content analysis, investigates on structure, coverage, and contents of television news in Colombia. Predominance of plain news, without context or with insufficient context elements, ends up in blurring of the guiding function of the journalism.
\end{abstract}

Key words: television, news, information, contextualization. 


\section{Introducción}

Lograr que los habitantes de un país tengan una actitud cívica activa y participen en las decisiones que sus dirigentes toman sobre los asuntos que les afectan es una tarea en la cual los medios de comunicación cumplen una función esencial. Como bien señala Acevedo (2005), es imposible que la gente opine sobre las decisiones y ordenamientos gubernamentales si no sabe nada sobre el proceso de decisión y en qué se sustentó, de manera que la información posibilita un ejercicio más pleno de los derechos de participación política para beneficio de la sociedad en su conjunto.

En este sentido, el papel de los noticieros televisivos resulta especialmente importante, ya que son éstos, por lo general, la única herramienta que tiene la mayoría de personas para informarse de lo que ocurre en el mundo, pues el acceso a la prensa, revistas e Internet, suele estar supeditado a la capacidad económica y a los hábitos lectores de los consumidores, que acceden con mayor facilidad a las noticias a través de la radio y la televisión (Burton, 2002).

Según datos del Estudio General de Medios, I Ola 2009, en Colombia la televisión es el medio de comunicación con mayor audiencia, seguido por la radio, las revistas independientes y la prensa. Este estudio señala que el $94.1 \%$ de los colombianos ve televisión, el 69.1\% escucha radio, el $36.9 \%$ lee revistas independientes, el $28.8 \%$ lee prensa, el $19.9 \%$ lee revistas de prensa, el 27\% usa Internet y el 3.8\% va a cine. Así pues, los noticieros de televisión tienen la gran responsabilidad de proveer a sus televidentes una información veraz, que no sólo les permita saber lo que pasa en la actualidad sino entender las causas de los hechos y establecer las consecuencias derivadas de estos.

Si las personas no sienten que la información tiene "algo que ver" con sus vidas, seguramente no prestarán atención a los hechos relatados. De allí se deriva que la pretendida objetividad, lograda a través del registro escueto de los hechos, no resulta ser lo más recomendable si se quiere formar un ciudadano democráticamente activo. Por el contrario, la información requiere de con- texto, de la escogencia de un encuadre noticioso adecuado, de un lenguaje y una estructura narrativa que permitan explicar la realidad para que el televidente saque sus propias conclusiones y se forme una opinión propia, pero sustentada en argumentos.

Por esta razón, resulta de especial interés conocer cuál es el tratamiento informativo dado por los telediarios colombianos a las noticias en la franja de mayor audiencia, con el fin de establecer si cumplen con la función de dar a la ciudadanía los elementos que requiere para ejercer sus derechos. En este sentido, la Comisión Nacional de Televisión (CNTV) de Colombia, en convenio con la Asociación Colombiana de Universidades (ASCUN), abrió, entre el 6 y el 23 de octubre de de 2009, la "primera convocatoria a presentación de propuestas de observación de contenidos de programas de televisión", para la cual licitaron alrededor de una decena de universidades del país, entre las cuales fue seleccionada la Universidad de La Sabana, a través del Observatorio de Medios (OM) y en colaboración con el Centro Multimedial de la Universidad Eafit, para desarrollar la investigación "Análisis de la estructura, cobertura y contenidos de los noticieros de la televisión colombiana", algunos de cuyos resultados son materia de este artículo.

\section{Método}

Para la realización del estudio se tomó una muestra de 15 noticieros, distribuida de la siguiente manera: 1 noticiero local (Bogotá), 9 regionales y 5 de carácter nacional. En total fueron analizadas 3.857 notas periodísticas, contenidas en 154 emisiones de los noticieros (ver tabla 1). El análisis, según lo estipulado por la CNTV, se hizo del 16 al 30 de octubre de 2009 en la franja prime time (7:00 p.m. a 11:00 p.m.).

El proceso de sistematización de la observación de los diferentes noticieros se hizo mediante una matriz que recogió la información de tres grandes categorías de estudio (estructura, cobertura y contenido) y alrededor de 22 factores con sus respectivas variables, sustentados en el Sistema Valor Agregado Periodístico (VAP), 
Tabla 1. Distribución de la muestra de estudio

\begin{tabular}{|c|c|c|c|}
\hline \multicolumn{4}{|c|}{ Universo de noticieros analizados } \\
\hline Canales & Noticieros & No. Emisiones & No. Notas \\
\hline \multicolumn{4}{|l|}{ Noticiero local (Bogotá) } \\
\hline City TV & City Noticias & 15 & 384 \\
\hline \multicolumn{4}{|l|}{ Noticieros regionales } \\
\hline Canal Capital & Capital Noticias & 15 & 257 \\
\hline Tele Antioquia & Tele Antioquia Noticias & 12 & 387 \\
\hline \multirow{2}{*}{ Tele Café } & Telecafé Noticias & 10 & 22 \\
\hline & 1 A Noticias & 4 & 74 \\
\hline Televisión Regional de Oriente & TRO Noticias & 12 & 188 \\
\hline Tele Caribe & CV Noticias & 6 & 180 \\
\hline \multirow{2}{*}{ Tele Pacífico } & Noti5 & 11 & 145 \\
\hline & UniNoticias & 3 & 56 \\
\hline Canal 13 & Canal 13 Noticias & 11 & 186 \\
\hline \multicolumn{4}{|l|}{ Noticieros nacionales } \\
\hline Canal Caracol & Caracol Noticias & 15 & 404 \\
\hline Canal RCN & Noticias RCN & 15 & 426 \\
\hline \multirow{3}{*}{ Canal Uno } & Tele País & 11 & 415 \\
\hline & CME & 11 & 423 \\
\hline & Noticias Uno & 4 & 112 \\
\hline Totales & 15 & 154 & 3857 \\
\hline
\end{tabular}

Fuente: elaboración propia.

validado en varios países por el equipo de investigadores de la Facultad de Comunicaciones de la Universidad Católica de Chile (Alessandri, F. et al., 2001). A través de este modelo se han determinado criterios medibles de calidad periodística que permiten establecer las características de la información publicada por un medio de comunicación y valorarlas.

Un segundo referente para el planteamiento de las categorías de análisis y sus correspondientes variables fue la experiencia del Observatorio de la Televisión de la Universidad Austral en Argentina (Delbosco, P. et al, 2006), que se ha preocupado por identificar específicamente la calidad de la información televisiva. Desde esta perspectiva la investigación empleó parte de sus lineamientos en materia de caracterización de los actores del proceso informativo y del lenguaje usado por los mediadores. La técnica utilizada fue el análisis de contenido.

\section{La supremacía de la noticia}

Uno de los hallazgos iniciales de la investigación es que los noticieros de televisión colombianos privilegian la noticia, entendida ésta como la primera aproximación a los hechos, el "qué" del acontecimiento. La profundidad del tratamiento informativo está generalmente ligada al género que el periodista escoge para presentar la información. La noticia, aunque puede estar muy bien contextualizada, se presta menos para la interpre- 
tación y la profundización que otros géneros como el reportaje, la crónica e, incluso, la entrevista. De esta manera, el hecho de que el género predominante en los noticieros de televisión sea la noticia (93\%) da cuenta de que es poco el esfuerzo por orientar a la opinión pública.

La especulación o análisis es la base informativa en apenas el $2 \%$ de las ocasiones, mientras que la base fáctica, es decir, contar los hechos como ocurrieron, el registro simple de la realidad, corresponde al 95\% de las informaciones publicadas. No por nada, el $71 \%$ de la estructura narrativa de las piezas informativas corresponde a la pirámide invertida, la más tradicional de las estructuras, muy propia de la noticia, y que privilegia los datos básicos de la información (qué, quién, cuándo, cómo, dónde). El contexto, por qué, para qué, con qué consecuencias, es pocas veces presentado en este tipo de estructura.

Pensamos, siguiendo a Miralles (2002), que la supremacía de la noticia en las agendas de los noticieros evidencia que los medios siguen aún inmersos en el "dogma y ritual" que en la prensa liberal ha originado la técnica de la pirámide invertida, en la cual se jerarquizan los datos de mayor a menor importancia, pero dejando de lado elementos de contexto y análisis necesarios para la comprensión de la realidad, es decir, para responder a un principio que ha hecho carrera en el periodismo y que es base fundamental del objetivismo: el de darle a la información la condición de "espejo de la realidad".

\section{Un marco "aséptico"}

Los medios de comunicación tienen no sólo la capacidad de imponer la agenda de temas sobre los cuales una sociedad opina y que jerarquiza como importantes, sino que también pueden decirle a la gente cómo pensar sobre esos temas o hechos registrados, según los atributos que destacan de un hecho informativo. Es lo que se conoce como segundo nivel del proceso de Agenda Setting, relacionado con los modelos del News Framing y del Priming, que reconocen la potencialidad de los medios para enmarcar los hechos desde una cierta perspectiva (Scheufele y Tewksbury, 2007).
Las investigaciones de Valkenburg, Semetko y De Vreese (1999), y Tewksbury et al. (2000), entre otros autores, han señalado que la manera en que se enfoca un tema influye en la percepción que el público desarrolla sobre ese asunto. En este sentido, se reafirma el hecho de que los noticieros colombianos se limitan al registro de lo que ocurre, sin interpretar o analizar, ya que el encuadre que predomina (64\%) es el informativo-descriptivo, es decir, aquel que sólo cuenta lo que ha pasado, sin destacar unos elementos en particular. Le siguen los encuadres de atribución de responsabilidades y de interés humano, cada uno con un $11 \%$, los cuales se centran, bien sea en señalar culpables o responsables de los hechos narrados, o en la vivencia humana, la personalización de una situación con la cual se espera que el televidente se identifique.

Al cruzar los encuadres con las temáticas, encontramos una alta asociación entre estas dos variables (Coef. de contingencia $=0.64 ; p=0.00$ ). Resalta, por ejemplo, que los medios tratan de establecer una especie de lejanía o pretendida objetividad a través del encuadre descriptivoinformativo, pero en los temas políticos hay una prevalencia del enfoque de conflicto, mientras que los judiciales son tratados muchas veces desde la asignación de responsabilidades, el conflicto y el interés humano. Como era de esperarse, el encuadre de Ganancias y/o pérdidas se presentó con más frecuencias en los temas económicos, tal como se observa en la tabla 2.

Varias investigaciones, como las de Capella y Jamieson (1997), Patterson (1994) y Semetko y Valkenburg (2000), han identificado un grupo de frames que son frecuentemente utilizados en noticias de política y economía, y que corresponden a lo hallado en la investigación realizada en los noticieros colombianos. Así, el encuadre de conflicto, que se presenta en los temas políticos, enfatiza en la controversia y el desacuerdo, mientras que el encuadre de ganancias y pérdidas enfatiza en las consecuencias que trae el hecho sobre la nación o sus habitantes. Tanto en Europa como en Estados Unidos, los resultados han sido similares (Bennet, 1995; Patterson, 2002; De Vresse, Jochen y Semetko, 2001), 
Tabla 2. Frecuencias cruzadas entre las temáticas de la pieza informativa y los diferentes de énfasis o encuadres.

\begin{tabular}{|c|c|c|c|c|c|}
\hline \multirow[b]{2}{*}{$\begin{array}{c}\text { Temática de la pieza infor- } \\
\text { mativa }\end{array}$} & \multicolumn{5}{|c|}{ Encuadre/énfasis } \\
\hline & $\begin{array}{l}\text { Ganancias- } \\
\text { Pérdidas }\end{array}$ & Interés Humano & Conflicto & $\begin{array}{c}\text { Asignación de } \\
\text { Responsabili- } \\
\text { dades }\end{array}$ & $\begin{array}{l}\text { Describir-Infor- } \\
\text { mar }\end{array}$ \\
\hline Política & 22 & 12 & 111 & 57 & 298 \\
\hline Judicial & 9 & 67 & 80 & 208 & 222 \\
\hline Economía & 185 & 17 & 3 & 8 & 99 \\
\hline Deportes & 1 & 18 & 5 & 13 & 512 \\
\hline Entretenimiento & 3 & 34 & 0 & 1 & 463 \\
\hline Internacional & 7 & 24 & 36 & 9 & 204 \\
\hline Ciencia y Tecnología & 1 & 4 & 0 & 1 & 37 \\
\hline Otros & 25 & 247 & 43 & 121 & 650 \\
\hline Total & 253 & 423 & 278 & 418 & 2485 \\
\hline
\end{tabular}

Fuente: Datos del Estudio

lo cual permite inferir la importancia de estos dos encuadres (conflicto, y ganancias y pérdidas), en lo que se refiere a la práctica periodística. Es de recordar que el conflicto es uno de los valores noticia que ha perdurado en la selección de lo que los medios publican y que las consecuencias económicas están relacionadas con los valores de proximidad y consecuencias para la nación, preponderantes en el proceso de gatekeeping o selección noticiosa.

El estudio de De Vreese (2004) muestra que los frames o encuadres en las noticias de televisión tienen la habilidad de dirigir los pensamientos de los televidentes hacia el elemento de la noticia que se ha destacado, por ejemplo, quienes han visto la noticia sobre la integración de la Unión Europea contada desde las posibles consecuencias económicas del hecho tienden a relatarlo desde ese mismo punto de vista, mientras quienes lo han visto contado desde las fricciones políticas que genera lo refieren como un hecho conflictivo e incluyen más puntos de vista negativos que quienes lo ven desde las consecuencias económicas.

La asignación de responsabilidades y el interés humano, marcos muy ligados a los temas judiciales, parten de la lógica de la espectacularización de la información, como lo explica Bourdieu (2000), quien afirma que el principio que guía la selección de la información en los noticieros televisivos es la búsqueda de lo sensacional y espectacular, con tendencia al drama.

\section{Más allá de la información}

Las noticias que transmiten los medios van más allá de configurar una "transmisión informe de datos", porque producen conocimiento, lo cual demanda del público que las recibe una capacidad cognitiva (Humanes y Ortega, 2000).

Ese conocimiento, que en últimas revela un conjunto de representaciones simbólicas de la sociedad a la cual se informa, está determinado por la calidad y profundidad de las informaciones que los medios ponen en la agenda pública.

Si en su mayor parte esas informaciones carecen de elementos que les dan profundidad, como el análisis y la interpretación, puede inferirse que el papel de los medios en busca de construir ese conocimiento colectivo sobre la realidad social dista de un ideal periodístico, especialmente desde una mirada ética.

Un periodismo más analítico e interpretativo frente a los asuntos públicos puede guiar a la sociedad a tomar mejores decisiones, no sólo en tiempos electorales, sino en el día a día.

Sin embargo, tal como señala Digón-Regueiro (2005), 
... el género del telediario, con sus rutinas de producción, su estructura rígida e invariable, su necesidad de simplificar y sintetizar gran cantidad de información en el tiempo establecidos y su dependencia de las audiencias y la publicidad, está más sujeto a las leyes del espectáculo que a la exigencia de un análisis riguroso y profundo de la realidad... Se busca más entretener y provocar emociones que ofrecer una explicación adecuada de los acontecimientos, que no niegue su complejidad, que remita a las causas y a los contextos en los que estos sucesos se producen.

Para que una información sea verdadera y significativa requiere de rigor, profundidad y claridad. Es necesario el contexto, que da sentido al hecho escueto y revela su verdadera significación. Vale entonces la pena recordar que según Gabriel Galdón López (1994, p. 161)

... el dar la verdad necesaria contextualizada y explicada y con sentido significa que hay que situarla en un contexto espacio-temporal; apuntar su significación en el devenir histórico; suministrar una información centrada en el proceso y no en los acontecimientos aislados; prever, mediante el conocimiento del pasado, el análisis del presente y la proyección en el futuro; tener en cuenta los procesos ideológicos relacionados con los acontecimientos; explicar el sentido que las cosas tienen por sí y por su repercusión en nosotros..."

Contextualizar es, pues, dar información adicional que permita al público tener un conocimiento general sobre antecedentes del hecho y conocer o inferir el posible desarrollo e, incluso, avizorar soluciones a una problemática determinada a partir de experiencias previas registradas en otros lugares o momentos históricos. De esta manera, la realidad se presenta con sus diversas aristas y el televidente puede entenderla con mayor facilidad y relacionarla con sus propias vivencias, de manera que no ve los hechos desconectados de su propio contexto y es posible que eso le mueva a tomar decisiones o acciones concretas.
En este aspecto, a los noticieros colombianos no les va del todo mal. Un 53\% de las notas periodísticas analizadas cuenta con información de contexto. Sin embargo, no todos los elementos del contexto están presentes en todos los casos: un $47 \%$ incluye antecedentes; un 19\%, consecuencias, y un $10 \%$, soluciones. Lo que se infiere es que los medios buscan que la gente entienda la causa de los hechos, pero dan poca importancia a las consecuencias que se podrían desprender de los mismos y a las soluciones que se podrían aportar. En este caso, es posible que los ciudadanos sientan que la información de tipo político o macroeconómico o, incluso alguna sobre salud o educación, no les toca directamente y pasen por encima de ella sin reflexionar en que, eventualmente, se verán afectados por esos acontecimientos o noticias.

Por otra parte, tal como lo señala GutiérrezCoba en una investigación sobre el tratamiento a la información judicial en la prensa (2001) no siempre los datos de contexto agregan valor a la información, debido a un uso inadecuado de los mismos: el contexto permite al lector formarse una idea clara de lo que ha sucedido y de las implicaciones futuras que podrían generarse a partir de tales acontecimientos, pero si tenemos en cuenta que esos elementos de contexto han sido, en su mayoría, formulados como parte de las noticias, es posible pensar que los medios prefieren presentar los antecedentes y las consecuencias como simples datos de registro, pero no se atreven a hacer un análisis orientador propio, dejando en los lectores la responsabilidad de formarse su propia opinión de un tema que, por tener cierto grado de especialización (términos e implicaciones jurídicas), requeriría una mayor ayuda por parte de expertos para ser entendido por quienes no son cercanos al tema (p. 78).

Otro hallazgo de la investigación es que los canales locales suelen dar más cabida a posibles soluciones como información de contexto, de manera que se orientan más a movilizar a las personas hacia actitudes proactivas. Los antecedentes o causas de los hechos tienen mayor presencia en los canales nacionales, mientras las 
consecuencias son tratadas en rangos similares por los tres tipos de canales (ver tabla 3).

\section{Conclusiones}

Esta investigación abordó el análisis de contenidos desde el campo de la producción de información, mas no desde la recepción, por lo que afirmar que las audiencias no cuentan con los elementos informativos y de análisis suficientes se desprende de una mirada inferencial, sobre todo por lo siguiente:

1. El hecho de que se privilegie la mera información y se deje a lugares secundarios el análisis y la interpretación deja ver que no existe un esfuerzo notorio de los medios analizados por distanciarse del periodismo puramente objetivista que, como lo dice Carlos Soria (1987, p. 62), obliga a muchos periodistas a ver los hechos "a través de unos cristales completamente transparentes que carecen de graduación para profundizar, ampliar y acercar la realidad a los ojos de un público al que se ofrece una visión chata del mundo, sin relieve, peso, ni proporciones".

2. Creer que con sólo informar se genera opinión pública es un error. La ciudadanía requiere orientación para asumir roles activos. Se necesita la identificación de temas comunes, el debate y la generación de corrientes de opinión. Miralles (2002, p. 45) afirma que "un modelo montado solamente sobre la idea de informar lo que sucede no proporciona las condiciones para conectar esos temas con los intereses del ciudadano, entre otras cosas, porque este se involucra por medio de valores, emociones, opciones y también de datos, pero de manera más secundaria".

3. La prevalencia de encuadres de conflicto en las noticias políticas tiende a reforzar en los televidentes la imagen negativa sobre este tema, mientras que aquellos temas tratados desde el encuadre de interés humano cruzan muchas veces el umbral de la identificación para llegar al del sensacionalismo y la exacerbación morbosa de los sentimientos de la gente.

4. El verdadero reporteo implica identificar tantos aspectos de un hecho noticioso como sea posible, y ponerlos en la agenda para su consideración, e identificar los asuntos más importantes del día y la agenda completa de atributos de esos asuntos, para que, eventualmente, estas informaciones puedan conducir a políticas públicas inteligentes y al desarrollo de opinión pública respecto del mejor curso de acción. Nada en esta tarea implica que los periodistas y los medios informativos abandonen su neutralidad. Es simplemente un llamado a que tomen responsabilidad sobre lo que hacen y lo realicen de un modo más reflexivo.

5. Lograr que los ciudadanos participen activamente en los procesos democráticos de opinión y toma de decisiones depende, en gran parte, de la labor de los noticieros de televisión, que deberían indagar en los intereses de sus públicos y en la necesidad de orientación que requieren

Tabla 3. Asociaciones significativas halladas entre la presencia de elementos del contexto y la cobertura legal del canal

\begin{tabular}{|c|c|c|c|c|c|c|c|c|}
\hline \multicolumn{9}{|c|}{ Cobertura legal del canal } \\
\hline Elementos del Contexto* & Local & $\%$ & Regional & $\%$ & Nacional & $\%$ & $\begin{array}{c}\text { Coef. de Con- } \\
\text { tingencia }\end{array}$ & $p$ \\
\hline Antecedentes & 179 & 43,9 & 664 & 50,6 & 958 & 60,4 & 0.14 & 0.00 \\
\hline Consecuencias posibles & 148 & 36,3 & 452 & 34,3 & 513 & 32,4 & 0.08 & 0.10 \\
\hline Posibles soluciones & 81 & 19,8 & 197 & 15 & 114 & 7,2 & 0.14 & 0.00 \\
\hline
\end{tabular}

* Se incluyen solamente los casos en que se registró presencia de elementos de contexto.

Fuente: Datos del Estudio 
sobre los temas fundamentales de la agenda noticiosa. Una investigación en este sentido, está todavía por desarrollarse.

\section{Referencias bibliográficas}

Acevedo, J. (2005). Derechos a una comunicación para todos. Lima: Veeduría Ciudadana de la Comunicación Social.

Alessandri, F. et al. (2001). VAP: un sistema métrico de la calidad periodística, en Cuadernos de Información 14, 112-120.

Asociación Colombiana para la Investigación de Medios-ACIM. (2009) Estudio general de medios, I Ola. Bogotá: Acim.

Bennett, W. (1995). News: the politics o illusion. New York: Longman.

Bordieu, P. (2000). Sobre la television. Barcelona: Anagrama.

Burton, G. (2002). More than meets the eye. An introduction to media studies. London: Arnold.

Capella, J. E Jamieson, K. (1997). Spiral of Cynicism: The Press and the Public Good. Nueva York: Oxford University Press.

De Vreese, C., Jochen, P. E Semetko, H. (2001). Framing politics at the launch of the Euro. A cross-national comparative study o frames in the new". Political Communication 18, 107-122

De Vreese, C. (2004). The effects of frames in political television news on issue interpretation and frame salience. Journalism and mass communication quarterly, vol. 81, No. 1, (spring 2004), 36-52.

Del Bosco, P. et al. (2006). Calidad televisiva. Tendencias y valores en la programación argentina. Buenos Aires: Universidad Austral.
Digón-Regueiro, P. (2005) Las otras noticias y los telediarios españoles: una propuesta de análisis. Comunicar 25, CD.

Galdón, G. (1994). Desinformación. Métodos, aspectos y soluciones. Pamplona: Universidad de Navarra.

Gutiérrez-Coba, L. (2001). La justicia ignorada: tratamiento de la información judicial en la prensa. Palabra-Clave 10, 67-83.

Humanes, M.L. E Ortega ,F. (2000). Algo más que periodistas. Sociologìa de una profesión. Barcelona: Ariel.

Miralles, A. (2002). Periodismo, opinión pública y agenda ciudadana. Bogotá, D. C.: Grupo Editorial Norma.

Patterson, T. (1994). Out of order. New York: Vintage.

Patterson, T. (2002). The vaninshing voter: public involvement in an age of uncertainty. Nueva York: Alfred A. Knopf.

Semetko, H. y Valkenburg, P. (2000). Framing european politics: a content analysis of press and television news. Journal of communication 50, No. 2, 93-109.

Soria, C. (1987). Prensa, paz, violencia y terrorismo. Pamplona: Universidad de Navarra.

Scheufele, D \& Tewksbury, D. (2007). Framing, Agenda Setting, and Priming: The Evolution of Three Media Effects Models, Journal of Communication 57, 9-20.

Valkenburg, P. M, Semetko, H. A. \& de Vreese, C. H. (1999). The effects of news frames on readers' thoughts and recall. Communications Research, 26, 550-569

Tewksbury et al. (2000). The interaction on news and advócate frames: manipulating audience perceptions of local public policy issue. Journalism and mass communication quarterly, 77 (4), 804-829. 